\title{
Efficacy and Safety of Fecal Microbiota Transplantation for the treatment of Clostridium difficile Infection in Patients with Liver Cirrhosis: a single center experience
}

\author{
Dana Alhaffar, BS ${ }^{1}$, Emmalee Phelps, BS $^{2}$, Monika Fischer, MD $^{3}$ \\ ${ }^{1}$ Indiana University School of Medicine, ${ }^{2,3}$ Indiana University School of Medicine, \\ Department of Gastroenterology/Hepatology \\ Background and Hypothesis: Fecal microbiota transplant (FMT) is an effective \\ therapy approaching a 90\% success rate for recurrent and severe CDI. However, \\ patients with liver cirrhosis are generally excluded from FMT trials due to \\ concerns of infectious complications. We aimed to investigate the efficacy and \\ safety of FMT in these patients.
}

Project Methods: Electronic medical records (Cerner) and an institutional FMT database (REDCap) were utilized to gather data on patient demographics, medical history, and follow-up. Inclusion criteria were the diagnosis of liver cirrhosis and FMT to treat CDI. The primary outcomes were FMT success at 8 weeks and adverse events within 12 weeks post-FMT.

Results: Among the 267 patients in the database, 15 had liver cirrhosis. Among these, $47 \%(N=7)$ were female. The average age was 61 years (range 28-83) and they received FMT between 2014 and 2017. There were 12 (80\%) patients with recurrent $C D I$ and $3(20 \%)$ with severe $C D I$. Eleven patients had decompensated cirrhosis; Child-Pough scores were $A: N=4, B: N=8$, and C: $N=3$. Five (33\%) failed before 8 weeks and needed 1-3 additional FMTs for cure. Fourteen (93.3\%) patients experienced adverse events. Five patients experienced unrelated SAEs (Table).

Conclusion: Patients with liver cirrhosis failed FMT more often than non-cirrhotic historical controls. While numerous AEs and SAEs were reported, few of the AEs were possibly related to the FMT and none of the SAEs were FMT-related. Importantly, no infectious complications were observed. Larger, multicenter studies are needed to establish efficacy and safety of FMT in cirrhotics before it can be recommended for widespread use. 